\title{
Métodos empíricos de la investigación
}

\author{
Empirical research methods
}

\begin{abstract}
Aileen A. Hernández-Rodríguez ${ }^{b}$, Virginia Argüelles-Pascual ${ }^{a}$, Raúl H. Palacios $^{c}$
Abstract:

In this work a graphic description of the empirical methods used in scientific research is presented. In the graphical representation, the classification of fundamental and complementary empirical methods is mentioned. Subsequently, the instruments that allow the researcher to capture relevant information, such as observation, experimentation, interview and survey as a scientific research technique are described.
\end{abstract}

\section{Keywords:}

empirical methods, research, interview, survey

\section{Resumen:}

En este trabajo, se presenta una descripción gráfica de los métodos empíricos usados en la investigación científica. En la representación gráfica, se menciona la clasificación de métodos empíricos fundamentales y complementarios. Posteriormente se describen los instrumentos que permiten al investigador captar información pertinente, tales como, observación, experimentación, entrevista y encuesta como técnica de investigación científica.

\section{Palabras Clave:}

métodos empíricos, investigación, entrevista, encuesta

\section{Introducción}

Los métodos empíricos se basan en la experiencia en el contacto con la realidad; es decir, se fundamentan en la experimentación y la lógica que, junto a la observación de fenómenos y su análisis estadístico, son los más utilizados en el campo de las ciencias sociales y en las ciencias naturales [3].

En este trabajo se representa gráficamente una breve descripción de los métodos empíricos usados en la investigación científica.

En primer lugar, se hace una clasificación dando como resultado los métodos empíricos fundamentales y por otro lado, los métodos empíricos complementarios. Así mismo, los primeros se dividen en dos tipos: la observación y la experimentación. $Y$ los segundos se clasifican en dos tipos: la encuesta y la entrevista.

La observación, como técnica de investigación científica, es un proceso riguroso que consiste en la percepción directa del objeto de investigación y permite conocer, de forma efectiva, el objeto de estudio para luego describir y analizar situaciones sobre la realidad estudiada [3]. La experimentación es un método empírico de estudio de un objeto, en el cual el investigador crea las condiciones necesarias 0 adecua las existentes, para el esclarecimiento de las propiedades o relaciones del objeto, que son de utilidad en la investigación [3].

Por otro lado, la encuesta es un método empírico complementario de investigación que supone la elaboración de un cuestionario, cuya aplicación masiva permite conocer las opiniones y valoraciones que sobre determinados asuntos poseen los sujetos (encuestados) seleccionados en la muestra [4].

En cuanto a la entrevista, es una técnica que consiste en recoger información mediante un proceso directo de comunicación entre entrevistador(es) y entrevistado(s), en

\footnotetext{
a Autor de Correspondencia, Universidad Autónoma del Estado de Hidalgo, Email: apascual@uaeh.edu.mx

b Universidad Autónoma del Estado de Hidalgo, Email: he333877@uaeh.edu.mx

c Universidad Autónoma del Estado de Hidalgo, Email: raul_palacios@uaeh.edu.mx
} 
el cual el entrevistado responde a cuestiones, previamente diseñadas en función de las dimensiones que se pretenden estudiar, planteadas por el entrevistador [3].

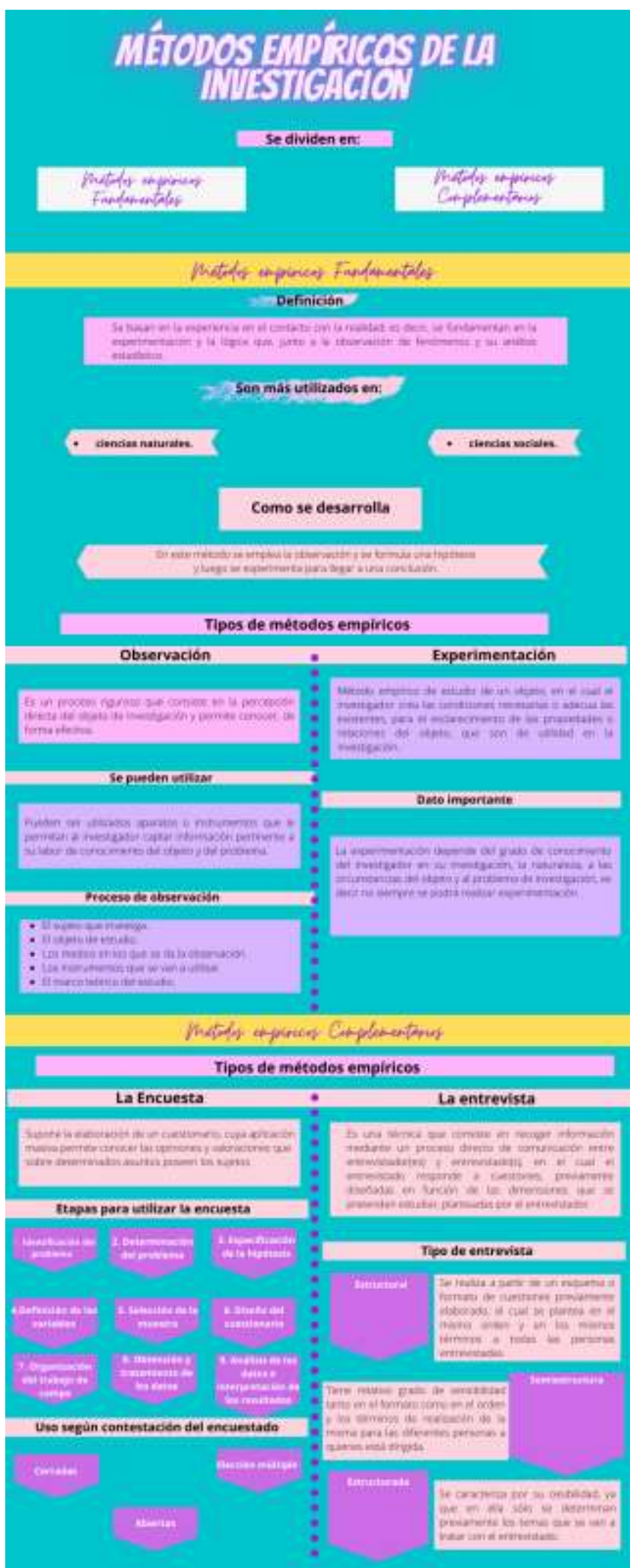

\section{Referencias}

[1] Hernández Sampieri, R. METODOLOGIA DE LA INVESTIGACION. Quinta edición, año 2010.

[2] Sampieri, R. H., Collado, C. F., \& Lucio, P. B. (1991). Metodologia de la investigación.

[3] Bernal, C. A. (2010). Metodología de la investigación: administración, economía, humanidades y ciencias sociales. (3 Edic.). Colombia: Prentice Hall.

[4] Anguita, J. C., Labrador, J. R., Campos, J. D., Casas Anguita, J., Repullo Labrador, J., \& Donado Campos, J. (2003). La encuesta como técnica de investigación. Elaboración de cuestionarios y tratamiento estadístico de los datos (I). Atención primaria, 31(8), 527-538. 